\title{
Audit of necropsies in a British district general hospital
}

\author{
M D Harris, J W Blundell
}

\begin{abstract}
A comprehensive study was made of the value of necropsies carried out over a six month period in Peterborough Health Authority, using a modification of the format proposed by Schned et al in Vermont, United States of America. Cause of death, clinical correlation, clinical factors contributing to death and clinical evaluation of the necropsy were recorded. The principal diseases were cardiac $(33 \%)$, and neoplastic $(29 \%)$. The pathologist disagreed with the clinical cause of death in $13 \%$ of cases and major unsuspected diagnoses were found in $30 \%$. Seventy nine per cent of necropsies provided additional information, including feedback on clinical investigations. Clinicians' questions were answered fully in $87 \%$. Necropsies were rated as "very valuable" in $44 \%$ of cases, "valuable" in $54 \%$, and "of no value" in only $2 \%$. In $14 \%$ of cases the clinical consultant stated that the results of the necropsy would affect future clinical practice.
\end{abstract}

These findings underline the essential place of necropsies in both audit and postgraduate education. The format described could conveniently be used by other pathology departments as part of an audit program.

As early as 1912, Cabot recognised the value of the necropsy in detecting common errors in clinical diagnosis. ${ }^{1}$ Several more recent studies have confirmed its merit in modern medical practice. $^{2-12}$ All have shown that, despite many advances in diagnostic techniques, a significant number of diagnoses are either not confirmed at, or not suspected until, necropsy. Although categories and criteria differ among studies, a major disagreement in diagnosis has been recorded in up to $29 \%$ of cases. ${ }^{13}$ Despite this evidence, the necropsy rate in most hospitals remains disappointingly low. ${ }^{14}$ With current pressures towards the participation of clinicians in audit, the place of the necropsy as the final arbiter of clinical performance is vital. ${ }^{15} 16$ Not only will such changes affect teaching hospitals, but also district general hospital practice. To establish the necropsy's place in peripheral hospital audit schemes it is important to determine how the figures for diagnostic errors in a district hospital compare with those found in the larger teaching hospitals. As pathologists will find themselves the subject of audit scrutiny, as well as providing information regarding clinical performance, it is also necessary to ask whether clinicians see the procedure as fulfilling their needs and how they perceive its value.

Over a six month period, we examined all hospital necropsies performed on adult patients dying within the Peterborough District Health Authority region, in a fairly typical district general hospital pathology department. We used as a framework for our study a modified version of the quality assessment program proposed by Schned et al in Vermont, United States of America. ${ }^{17}$ Our findings are of particular interest as they reflect British district general hospital practice, previous studies having concentrated on teaching hospitals.

\section{Methods}

Peterborough District Health Authority comprises two main units, Peterborough District Hospital (PDH), a 392 bed general hospital where the mortuary is situated, and its near neighbour, the Edith Cavell Hospital (ECH), with 275 beds. Both hospitals receive acute and routine admissions. Service is also provided to several small outlying hospitals. All necropsies performed on adult patients dying in PDH or ECH, between 8 January and 6 July 1990, were included in the study. Coroners' necropsies, together with paediatric or perinatal cases, were excluded, as were deaths occurring in the outlying hospitals.

Clinicians requesting necropsies were asked to provide general clinical information, state the cause of death, as recorded on the death certificate, and any specific questions to be answered by the necropsy (Part A) (figure). As is routine practice, this part was usually completed by the house officer or senior house officer. Part B of the form was completed after the necropsy. In this, the pathologist was asked to state how he or she would certify the cause of death, to record the presence of any important additional pathology, and whether the findings of the necropsy agreed, partly agreed, or disagreed with the cause of death stated in Part A.

Shortly afterwards the consultant in charge of the case was asked to complete Part $\mathrm{C}$ of the form. Several questions were asked, including performance assessment of the necropsy. To ensure a $100 \%$ response and uniformity in interpretation of the questions all forms, along with the necropsy report and clinical notes, were personally taken to the clinician. Clinicians were encouraged to be honest in their

\section{Hincopathology, Hinchingbrooke Park, Huntingdon Cambridgeshire PE18 8NT Accepted for publication 22 May 1991 \\ Distopathology and Morbid Anatom Hospital, Cambridg Department of Peterborough District Hospital, J W Blundell}


Request for necropsy examination

PART A (To be completed for all hospital necropsies)

NAME OF PATIENT:

AGE:

DATE OF BIRTH:

CONSULTANT IN CHARGE:

DATE, TIME AND PLACE OF DEATH:

ANY SPECIAL RISKS, eg. AIDS, hepatitis, TB, radioactive isotopes, etc?

CLINICAL SUMMARY:

What cause of death have you put on the death certificate? Other findings anticipated? Are there any specific questions you wish answered by the necropsy? COMMENTS:

PART B (To be completed by the pathologist after the necropsy)

Cause of death: Other incidental findings:

(If cause of death not found, or awaiting further tests, please specify)

Degree of agreement between causes of death in A \& B-AGREE/PARTLY AGREE/DISAGREE COMMENTS:

PART C (To be completed by the clinical consultant in charge after the necropsy)

Do you generally agree with the pre-necropsy assessment of cause of death? YES/NO

Were the results of any tests confirmed or refuted? YES/NO

Did any iatrogenic factors or unsuspected diagnoses contribute to death? YES/NO

Did the necropsy answer your questions? YES/PARTLY/NO

How do you rate the overall value of this necropsy? VERY VALUABLE/SOME VALUE/NO VALUE

Will the results of this necropsy change your future practice? YES/NO

COMMENTS:

appraisal. The forms were analysed in accordance with a modification of the four main categories proposed in the Vermont study. The major difference in our study is the expansion of the section dealing with the clinician's assessment of the necropsy.

These categories were as follows:

I Underlying disease and cause of death

Major disease categories, such as cardiac, neoplastic, and causes of death, such as pneumonia, cardiac failure, were recorded for each case by both clinician and pathologist. Although only one major disease category was allowed for each case, any number of conditions were recordable under cause of death.

\section{Clinical relevance of necropsy findings}

Each necropsy was classed as either showing a major disagreement in diagnosis (group 1), confirming the main diagnosis (group 4), or offering no contribution to diagnosis (group 5). Each case was also assigned to any, all, or none of the remaining groups.

III Clinical factors contributing to death In each case death was classified as due entirely to the major disease diagnosed clinically, due to unsuspected conditions, or due to iatrogenic factors. Both the clinician's and pathologist's appraisals were recorded.
IV Performance assessment of the necropsy Necropsies were graded by the consultant as to whether questions were answered fully, partly, or not at all, and as very valuable, of some value, or of no value. The clinician was also asked whether the results would affect future practice. General comments, including whether the clinical consultant agreed with the pathologist's opinion, were also recorded in this section.

\section{Results}

The necropsy rate for the whole of 1990 , which covers the six month period studied, was $13 \%$ (996 deaths; 130 necropsies). This figure includes all adult hospital deaths and hospital necropsies for all of the hospitals served. Sixty three of these cases fulfilled the criteria for inclusion in this study.

\section{UNDERLYING DISEASE AND CAUSE OF DEATH} The major disease categories and conditions recorded under cause of death are summarised in table 1. Although the overall figures for disease incidence recorded before and after necropsy are similar for both major disease categories and causes of death, there was considerable disparity in individual cases. Of the 16 cases of bronchopneumonia diagnosed clinically, only 11 were confirmed at necropsy ( $31 \%$ overdiagnosis). A further three cases of bronchopneumonia were first diagnosed at necropsy ( $21 \%$ underdiagnosis). In all but three cases the consultant supported the house officer's certification of cause of death. 
Table 1 Death certification as recorded by pathologists and clinicians

\begin{tabular}{lrr}
\hline & Pathologists & Clinicians \\
\hline Major disease category & & \\
Cardiac & $21(33 \%)$ & $21(33 \%)$ \\
Neoplastic & $18(29 \%)$ & $14(22 \%)$ \\
Chronic lung disease & $6(10 \%)$ & $4(6 \%)$ \\
Neurological & $6(10 \%)$ & $5(8 \%)$ \\
Chronic gastrointestinal disease & $5(8 \%)$ & $6(10 \%)$ \\
Haematological & $4(6 \%)$ & $4(6 \%)$ \\
Other & $3(5 \%)$ & $9(14 \%)$ \\
Causes of death & & \\
Infection/septicaemia & $27(43 \%)$ & $20(32 \%)$ \\
Cardiac failure/arrhythmia & $16(25 \%)$ & $15(24 \%)$ \\
Pneumonia & $14(22 \%)$ & $16(25 \%)$ \\
Metastatic neoplasm & $8(13 \%)$ & $6(10 \%)$ \\
Myocardial infarct & $7(11 \%)$ & $8(13 \%)$ \\
Gastrointestinal bleed & $6(10 \%)$ & $2(3 \%)$ \\
Renal failure & $5(8 \%)$ & $6(10 \%)$ \\
Pulmonary embolus & $3(5 \%)$ & $5(8 \%)$ \\
Peritonitis & $3(5 \%)$ & $1(2 \%)$ \\
\hline
\end{tabular}

\section{CLINICAL RELEVANCE OF NECROPSY FINDINGS}

The degree of clinical correlation of the necropsy findings in this study is shown in table 2. Major disagreement in diagnosis was found in eight $(13 \%)$ cases. These were either cases in which death was due to unsuspected major diagnoses, or where the presence of the main disease diagnosed clinically could not be confirmed (table 3). Major unsuspected diagnoses were present in $19(30 \%)$ cases (table 4$)$. The presence of pathology relating to the main diagnosis was confirmed in $54(86 \%)$ cases but this was judged by the pathologist not to be the main cause of death in $14(22 \%)$ cases. In one case the clinician felt that the necropsy had made no clinical contribution to the case.

Important additional information was provided by the necropsy in $50(79 \%)$ cases. In $32(51 \%)$ cases this was in the form of minor unsuspected pathology, such as small pulmonary emboli. Forty three $(68 \%)$ necropsies provided feedback on the accuracy of diagnostic tests- $x$ ray pictures, computed tomograms, electrocardiographs. In $37(59 \%)$ cases the results of these tests were confirmed; in 11 $(18 \%)$ cases the necropsy findings disagreed with the results of investigations before death. In five cases necropsy confirmed some test results, but disagreed with others. These were included in both categories. In $20(32 \%)$ cases no relevant tests were done.

\section{CLINICAL FACTORS CONTRIBUTING TO DEATH}

In $12(19 \%)$ cases the clinician felt that unsuspected factors or diseases contributed to death. On reviewing the data, the pathologists considered that there was sufficient reason to assign a further $\operatorname{six}(9 \%)$ cases to this category. These cases are summarised in tables 5 and 6 . No iatrogenic conditions were considered by either party to have contributed to any death.

Table 2 Clinical relevance of necropsy findings

\begin{tabular}{ll}
\hline 1 Major disagreement in diagnosis & $8(13 \%)$ \\
2 Major unsuspected diagnosis & $19(30 \%)$ \\
3 Significant clarification of diagnosis & $40(63 \%)$ \\
4 Confirms presence of main diagnosis & $54(86 \%)$ \\
5 No contribution to diagnosis & $1(2 \%)$ \\
6 Significant additional information & $50(79 \%)$ \\
additional pathology & $32(51 \%)$ \\
feedback on tests & $43(68 \%)$ \\
\hline
\end{tabular}

Table 3 Cases with major disagreement in diagnosis

Acute bronchitis misdiagnosed as pulmonary emboli Ischaemic heart disease misdiagnosed as pulmonary emboli Bleeding duodenal ulcer misdiagnosed as pulmonary emboli Pulmonary emboli missed

Renal failure due to heart failure, renal arteries not involved in Renal failure due

aortic aneurysm

Missed ruptured aortic aneurysm

Perforated carcinoma of the stomach misdiagnosed as ischaemic heart disease

Carcinomatosis not confirmed (no carcinoma)

Table 4 Major unsuspected diagnosis

Gastrointestinal bleeding ( 2 cases)

Carcinoma of the ovary

Peritonitis due to perforated carcinoma of the colon

Carcinoma of the stomach

Carcinomatosis ( 3 cases)

Carcinoma of the bronchus

Pulmonary emboli

Cerebral abscess

Haemopericardium

Severe hypertensive heart disease

Bronchopneumonia ( 2 cases)

Perforated carcinoma of the stomach with liver metastases

Ruptured aortic aneurysm

Urinary tract infection

Subphrenic abscess

Table 5 Unsuspected factors contributing to deathcases identified by clinicians

Missed ruptured aortic aneurysm

Pneumonia misdiagnosed as cardiac failure

Missed myocardial infarct

Pulmonary absess and carcinoma of the ovary missed

Carcinoma of the stomach misdiagnosed as carcinoma of the rectum

Missed septicaemia in an immunosuppressed patient

Missed pulmonary emboli

Misjudged age of myocardial infarct, treated with

streptokinase, haemopericardium

Missed gastrointestinal bleeding ( 2 cases)

Overestimated the degree of chronic respiratory disease, didn't treat aggressively

Inguinal hernia misdiagnosed as secondary carcinoma

Table 6 Unsuspected factors contributing to deathadditional cases identified by pathologists

Misdiagnosed peritonitis as bowel obstruction
Recurrent, disseminated carcinoma of the bladder missed, but Recurrent, disse
not treatable

not treatable
Urinary tract infection missed as cause for diabetic

ketoacidosis

Bronchopneumonia missed ( 2 cases)

Perforated carcinoma of the stomach misdiagnosed as perforated duodenal ulcer

\section{PERFORMANCE ASSESSMENT OF THE} NECROPSY

In $55(87 \%)$ cases the clinician stated that the necropsy had fully answered the questions posed. Questions were partly answered in a further five $(8 \%)$ cases. In three $(5 \%)$ cases the clinician felt that questions were unanswered. Twenty eight $(44 \%)$ of the necropsies performed were felt to be very valuable, $34(54 \%)$ of some value, and only one $(2 \%)$ case was considered to be of no value. In nine $(14 \%)$ cases the consultant stated that the results of the necropsy would affect future practice. The clinicians' comments in these cases are summarised in table 7 . In two (3\%) cases the clinician disagreed with the pathologist's interpretation of the cause of death, although the actual findings at necropsy were not disputed. 
Table 7 Clinicians' comments on future modifications to management

Will put more faith in echocardiogram and treat infective endocarditis earlier

Will be less inclined to assume that symptoms are due to previously diagnosed malignancy

Will push gynaecologists to investigate pelvic pathology

(carcinoma of the ovary missed)

Will more readily investigate bowel symptoms even if barium enema negative

More diligent attempts to aspirate septic arthritis; will not trust orthopaedic opinion

More vigorous treatment of respiratory failure (degree of

More vigorous treatment of respi
chronic disease overestimated)

Assumed inguinal hernia to be lymph node secondary tumour, will biopsy in future

Will use angiography more readily in young ischaemic heart disease

Exposed fault in $\mathrm{x}$-ray report filing

Missed myocardial infarct; will be more suspicious in simila cases in future

\section{Discussion}

In our study the clinically attributed cause of death was completely wrong in $13 \%$ of cases and unsuspected major diagnoses were found in $30 \%$. In Schned's original study ${ }^{17}$ a major disagreement in principal diagnosis was recorded in only one (1\%) case, with $12 \%$ comprising unsuspected major diagnoses. Our figures, however, are comparable with the major discrepancy rates found previously. ${ }^{2-12}$ Hill and Anderson have summarised these data. ${ }^{13}$ Like Schned, we found that most necropsies (86\%) confirmed the presence of the major clinical diagnosis; the rate of indeterminate necropsies was very low $(2 \%)$. Clearly, our clinical colleagues regarded the necropsy as being of great importance, stating that $98 \%$ were of value, with $44 \%$ being "very valuable" Clinicians' question were answered fully in $87 \%$ of cases and partially in a further $8 \%$. In $14 \%$ of cases the consultant actually stated that future practice would be modified in the light of the findings. In a study published in $1989,{ }^{11}$ which also used Schned's proforma, Harrison and Hourihane found that $58 \%$ of their necropsies were considered helpful. A similar evaluation, undertaken in Aberdeen in $1977^{12}$ also showed that specific questions were answered in $83 \%$ of cases.

This study, which, to our knowledge is the first such analysis of the role of the necropsy in a British district general hospital, confirms that the clinicians' confidence in their armament of diagnostic tests is not justified, and that the rate of error is comparable with that encountered in teaching hospital studies. In the light of the above figures, which reflect a generally high degree of satisfaction with the necropsy there can be little doubt that the procedure represents a most valuable form of postgraduate education. With a considerable number of major and minor unsuspected diagnoses, as well as positive and negative feedback on ante-mortem tests, necropsies must form a cornerstone in any audit program.

We also tested a convenient proforma for data collection and audit on the performance of the necropsy itself. We recommend that other pathology departments should adopt this, or a similar protocol, as part of their own audit plan.

One point, which clearly emerged from our study, was the subjective nature of case assignment to each category. Although this can be standardised within an individual department, there may be problems when results from different centres are compared. We suggest that standardised categories and criteria should be agreed and used in future studies to facilitate national and international comparison of data.

1 Cabot RC. Diagnostic pitfalls identified during a study of three thousand autopsies. JAMA 1912;59:2295-8.

2 Britton M. Clinical diagnostics; experience from 383 autopsied cases. Acta Med Scand 1974;196:211-19.

3 Cameron HM, McGoogan E, Watson H. Necropsy: a yardstick for clinical diagnoses. Br Med J 1980;281:985-8.

4 Cameron HM, McGoogan E. A prospective study of 1152 hospital autopsies: I. Inaccuracies in death certification J Pathol 1981;133:273-83.

5 Cameron HM, McGoogan E. A prospective study of 1152 hospital autopsies: II. Analysis of inaccuracies in clinical diagnoses and their significance. J Pathol 1981;133 285-300.

6 Thurlbeck WM. Accuracy of clinical diagnosis in a Canadian teaching hospital. Can Med Assoc J 1981;125:443-7.

7 Goldman L, Sayson R, Robbins S, Cohn LH, Bettmann M, Weisberg $M$. The value of the autopsy in three medical eras. N Engl J Med 1983;308:1000-5.

8 Kircher T, Nelson J, Burdo $H$. The autopsy as a measure of accuracy of the death certificate. $N$ Engl J Med 1985; 313:1263-9.

9 Landefeld CS, Chren MM, Myers A, Geller R, Robbins S, Goldman L. Diagnostic yield of the autopsy in a university hospital and a community hospital. N Engl J Med 1988 318:1249-54.

10 Karwinski B, Hartveit F. Death certification; increased clinical confidence in diagnosis and lack of interest in confirmation by autopsy is not justified. J Clin Pathol 1989;42:13-17.

11 Harrison M, Hourihane D O'B. Quality assurance programme for necropsies. J Clin Pathol 1989;42:1190-3.

12 Fowler EF, Nicol AG, Reid IN. Evaluation of a teaching hospital necropsy service. J Clin Pathol 1977;30:575-8.

13 Hill RB, Anderson RE, eds. Missed diagnoses. Towards an understanding of diagnostic discrepancies. In: The autopsy-Medical practice and public policy. London Butterworths, 1988:67-90

14 Chana J, Rhys-Maitland R, Hon P, Scott P, Thomas C, Hopkins $A$. Who asks permission for an autopsy? $J$ Roy Coll Phys Lond 1990;24:185-8.

15 Hill RB, Anderson RE. Is a valid quality assurance programme possible without the autopsy? Hum Pathol $1988 ; 19: 1125-6$

16 Anderson RE, Hill RB, Gorstein F. A model for the autopsybased quality assessment of medical diagnostics. Hum Pathol 1990;21:174-81.

17 Schned AR, Mogielnicki RP, Stauffer ME. A comprehensive quality assessment programme on the autopsy service. $\mathrm{Am}$ J Clin Pathol 1986;86:133-8. 